\title{
Reflection on the Short Video's Algorithm-based Communication in the Age of Post-truth
}

Boyu Zhao*

Huazhong University of Science and Technology, Wuhan 430074, China. E-mail: zhaoboyu@ hust.edu.cn

Abstract: Combining the characteristics of post-truth era with short video's form, this article has made a new understanding of Walter Lippmann's "pseudo-environment". This author reconsiders the short video based on algorithm communication. In order to reduce the political risk of the algorithm, it is necessary to give full play to the advantages of the algorithm, improve the recommendation mechanism of the recommendation algorithm and take the algorithm as the fourth gatekeeper to check the information, according to the operation mechanism from the platform's point of view.

Keywords: Post-truth; Reflection on Algorithm; Short Video

\section{Significance of studying algorithm-based communication in post-truth era}

Oxford English Dictionary selected "post-truth" as a word of the year for 2016, in which post-truth was defined as "relating to or denoting circumstances in which objective facts are less influential in shaping public opinion than appeals to emotion and personal belief". Its basic structure is that "cocoons" of social relationship will lead to information cocoons, and in such situation, emotion and standing point is much more important than truth ${ }^{[1]}$. Meanwhile, the forms of traditional information dissemination have been fundamentally changed in the age of post-truth featured with fragmentation, decentralization, tribalization, and idolization of information.

Algorithm refers to accurate and complete descriptions and a series of clear instructions that are followed to solve a problem. It is also a strategy for finding solutions in a systematical way. The promotion of recommendation algorithm and recognition algorithm can not only make those tasks possible which needed expert's support or were too complicated to accomplish, but also save the decision-making cost and enhance political efficiency and scientificalness, objectivity of decision-making. Information cocoon is increasing in the age of post-truth, though the accuracy of political communication has been improved significantly through the combination of individuality and information recommendation $^{[2]}$. Algorithm communication recommends contents that are similar to receiver's taste and preference to cater to user side, taking the 2016 president election as an example, to call for positive participation of voters with the same political views and makes Trump win the election.

By its scientific and technological legitimacy, algorithm communication evade democratic supervision. Current algorithm has successfully blocked all kinds of social questions regarding its deviation from publicity, which makes people ignore risks behind. However, political risk of algorithmic communication is a modern bad result brought by the

Copyright (C) 2021 Boyu Zhao

doi: $10.18686 / \mathrm{mcs} . v 3 \mathrm{i} 1.1373$

This is an open-access article distributed under the terms of the Creative Commons Attribution Non-Commercial License

(http://creativecommons.org/licenses/by-nc/4.0/), which permits unrestricted non-commercial use, distribution, and reproduction in any medium,

provided the original work is properly cited. 
rapid development of technological reason. Therefore, it is urgent to uncover the essence of algorithmic and attach importance to the destruction of its risk to society in order to build a counterbalance mechanism.

\section{Function and influence of news videos}

The author selected news videos as keywords, searched them on the web of CNKI, and listed all the keywords of Chinese-written articles and some English-written ones from the year of 2009 to now. It was found that keywords, such as mobile, video news, short video, mobile internet are most frequently mentioned in the recent ten years. Moreover, these words are related to mobile short video. Thus, short video, as a new form of news expression, has attracted attention of many scholars in China.

Short video as an information spreading form emerged few years ago. Neil Postman once remarked that a new form of information spreading brings social changes which are not only confined to the content it conveys, more significantly, the new form has defined transmission speed, sources, amount and existing context of information, greatly influencing social relationship, structure and culture during a specific time. Short video has low using threshold, featured with functions of lightweight upload and receive, three-dimensional open sharing channels and personality creative content expression. It makes text, pictures in past social network transfer to videos, which caters to reading habits and social needs of people in current years.

Being more free to produce and upload news is the characteristic of spreading news through short video. While short video just needs one cell phone, there will be high production cost for traditional video news, for example, videoing equipment which are of high degree of difficulty to operate. Besides, short video has set limited shooting time which makes it an instant messaging, caters to consumption habits of lightweight and snackable content, and has higher user acceptance. Due to counting in seconds, short video has stronger user stickiness and it allows users to spend their time in fragment. Besides, short video news being clear point of view and concise content, it is easy to be accepted by consumers. Therefore, the impact of short video news on users is far-reaching, and far beyond the impact of text or television.

\section{Short video: To built a special pseudo-environment}

Walter Lippmann defines pseudo-environment as the environment depicted by mass media in his book Public Opinion. Pseudo-environment is not a mirror-like reflection of the objective environment, and cannot be equated with the objective environment. Instead, media provide people about the environment after selecting, processing and re-structuring events or information.

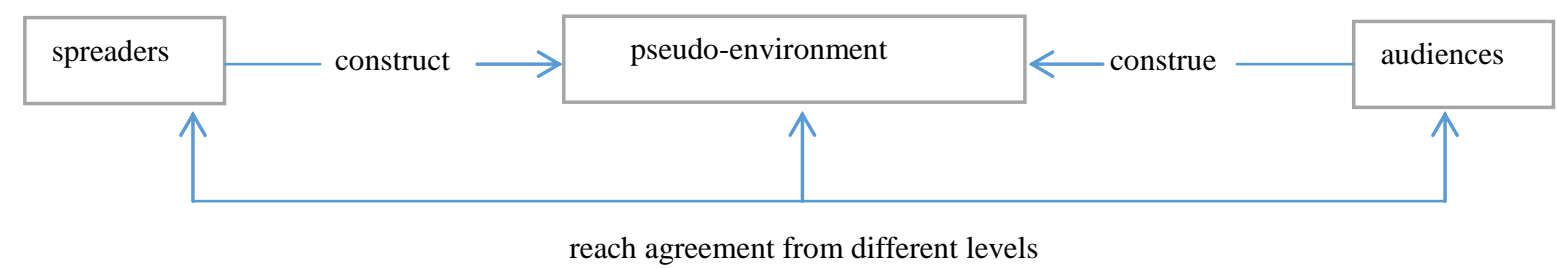

Figure 1. Consequences of long-term pseudo-environment.

Traditional pseudo-environment is an environment constructed by emphasizing the media's reporting on events. However, the pseudo-environment created by news spread on short videos is special. There left much less space for video's production compared with the description of words, which can eliminate the concealment of traditional media reports in theory. However, the fact is that its concealment will be more obvious. Video is more vivid, so it will lead people to think less about causes and consequences, and to be covered up by the "truth" they see.

Let's analyse a case and take it as an example: a female driver was beaten in Chengdu, China. On the afternoon of May 3, 2015, near the Jiaozi Overpass located in the third circuit of Chengdu, a female driver was forced to stop by the rear car driver and dragged out of her car. The male driver continuously hit the female driver's head and face, which 
caused the female driver's shoulder fracture, concussion and bruises. The video of the female driver being beaten was posted on the Internet, which led all netizens to condemn the male driver.

However, during the investigation, the police found through the records of the driving recorder of the male driver that before the female driver was beaten, she changed lanes several times to jam his car. When this video was posted on the Internet, everyone then began to condemn the female driver.

In this news, many ignored the cause and effect of the matter, only saw what was provided in front of them, and made a final conclusion on the matter. Whereas, in written news reports, people would think about why male drivers reach female drivers for no reason, even being road rage. However, short videos are highly acceptable that users think less. And with the support of algorithm, people will get in touch with a lot of relevant news, which is what people are more willing to believe. Internet users in the post-truth era are extremely emotional, and may lead to a conclusion based on "morality" before things are completely clear.

In the age of post-truth, under the reality based on algorithm communication, short videos have created a special pseudo-environment that seems more transparent, but actually more hidden.

\section{Political nature of algorithm communication}

Through the above case, it can be clearly learned that the short video based on algorithm communication can easy develop according to the communicator's idea. At the beginning of the article, it mentions that in the post-truth era, the promotion of recommendation algorithm and recognition algorithm can not only make those tasks possible which needed expert's support or were too complicated to accomplish, but also save the decision-making cost and enhance political efficiency and scientificalness. However, the combination of personalized recommendation and information dissemination has greatly improved the accuracy of political communication and intensified cocoon, which will make users believe in some of the "truth" they have seen.

The essence of the political system is to distribute value authoritatively for a society. Its important features are democracy, fairness, legitimacy, openness and transparency. The algorithm can achieve an idealized fairness and democracy, which leads to the strong political nature of algorithm propagation. However, since the US election in 2016, fake news pushed by social media based on improper algorithms has plagued the whole world, which not only tears the society, but becomes the ferment of political polarization. It can be seen that the risks of algorithmic politics include moral hazard, decision-making risk, legitimacy crisis and unexpected unknown risks caused by the inherent defects of algorithms when they are applied in the political field ${ }^{[3]}$. It also includes security risks caused by improper use of algorithms in the field of political communication (especially the proliferation of "fake" news caused by intelligent recommendation algorithms).

Therefore, it is necessary to make rational use of algorithm communication, seek advantages, avoid disadvantages, and give full play to its advantages.

\section{Methods for seeking advantages and avoiding disadvantages of algo- rithm communication}

The greatest victory in technology is almost juxtaposed with the greatest disaster. It can be easily understood that technology is a double-edged sword. So how can algorithm communication improve its strengths and avoid weaknesses to better serve politics?

The author starts with the operation mechanism of the algorithm, and looks for ideas to give full play to the advantages of the algorithm. The algorithm is based on a large amount of user data, and filters out the information that users like from a large amount of content, so that the platform can obtain more user traffic ${ }^{[4]}$.

Therefore, the article will analyze it from the following three perspectives: users, content and platform. From the perspective of users and content, the current research is relatively mature, such as improving the quality of users, making better content by content producers and so on. The article won't go into details, and will focus on the analysis from 
the perspective of platform.

The algorithm is spread on platforms. When designing algorithm, platforms should give full play to the role of the fourth gatekeeper of the algorithm, and seek advantages and avoid disadvantages ${ }^{[5]}$. Shoemaker divided gatekeeper theory and model into five categories:

(1) Personal level. It refers to the degree to which individuals are responsible for checking and choosing, which is composed of concepts such as personal explanation, decision-making, personality, background, values and roles;

(2) Routine level of media work. It refers to "modeled, step-by-step and repeated practice forms used by media personnel for work";

(3) Organizational levels. It includes internal factors under different organizations and different group decision-making modes;

(4) The level of social groups outside the media. It mainly focuses on the external characteristics of various organizations and their representatives (including market forces and political alliances) that influence the process of customs control;

(5) The level of social system. It explores the influence of ideology and culture on customs control.

Current platform pays too much attention to the traffic of the platform when designing algorithms. But for a mature platform, it bears more social responsibility, even political responsibility. The current recommendation algorithms are mainly based on content recommendation and collaborative filtering recommendation, as far as the author knows. When making recommendations, the platform should add some new filtering mechanisms ${ }^{[6]}$.

(1) Short video news will be accompanied by more comments, and there is a basic emotional attitude judgment for these comment if the overall comments are negative and irrational. Limit the recommendation of such videos, such as speeding up the attenuation mechanism;

(2) The identification mechanism of short video content is added, and a layer of filtering mechanism is added to the content with high content homogeneity, and the most authoritative publishers of these information sources with high content homogeneity are selected for exposure. This will not only promote the publishing source to produce more exclusive reports, but also solve the problem of stealing videos to some extent.

\section{References}

1. Peng L. Illusion, prisoner of algorithm, and transfer of rights: The new risks in the age of data and algorithm. Journal of Northwest Normal University (Social Sciences) 2018; (5): 20-29.

2. Quan Y, Chen L. Public deviation and subject distortion risk in the age of algorithm communication. Journal of Central China Normal University (Humanities and Social Sciences) 2019; (1): 149-156.

3. Ru X. Algorithmic politics: Risk, logic and governance. Journal of Xiamen University (Arts \& Social Sciences) 2018; (6): 27-38

4. Yin J, Liu Y. Research on innovation mode and countermeasure of short news video in China (in Chinese). Press Circles 2017; (12): 34-38.

5. Luo X. Structural defects: Reconsideration for the gatekeeping theory in the age of Internet. Journalism \& Communication 2011; (3): 68-76, 112-113.

6. Sheng Y. Application of short video sharing and innovation of news communication (in Chinese). News World 2014; (7): 187-188. 